Research Article

\title{
Simulation of J-Solution Solving Process of Navier-Stokes Equation
}

\author{
Wenjie Wang (iD) and Melkamu Teshome Ayana $\mathbb{1 D}^{2}$ \\ ${ }^{1}$ School of Science, Chang'an University, Xi'an 710064, China \\ ${ }^{2}$ Department of Hydraulic and Water Resources Engineering, Arba Minch University, Arba Minch, Ethiopia
}

Correspondence should be addressed to Wenjie Wang; wenjiew123@126.com

Received 12 March 2021; Revised 18 April 2021; Accepted 29 April 2021; Published 7 May 2021

Academic Editor: Vijay Kumar

Copyright (c) 2021 Wenjie Wang and Melkamu Teshome Ayana. This is an open access article distributed under the Creative Commons Attribution License, which permits unrestricted use, distribution, and reproduction in any medium, provided the original work is properly cited.

To avoid grid degradation, the numerical analysis of the j-solution of the Navier-Stokes equation has been studied. The Navier-Stokes equations describe the motion of viscous fluid substances. On the basis of the advantages and disadvantages of the Navier-Stokes equations, the incompressible terms and the nonlinear terms are separated, and the original boundary conditions satisfying the $j$-solution of the Navier-Stokes equation are analyzed. Secondly, the development of a computational grid has been introduced; the turbulence model has also been described. The fluid form and the initial value of the $j$-solution of the Navier-Stokes equation are combined. The original boundary conditions are solved by a computer, and the nonlinear turbulence equations are derived, which control the fluid flow. The simulation of the fine grid is comprehended to analyze the research outcome. Simulation analysis is carried out to generate multiblock-structured grids with high quality. The j-solution on the grid points is the $\mathrm{j}$-solution that can be used with a fewer number of meshes under the same conditions. The proposed work is easy to implement, and it consumes lesser memory. The results obtained are able to avoid mesh degradation skillfully, and the generated mesh exhibits the characteristics of smoothness, orthogonality, and controllability, which eventually improves the calculation accuracy.

\section{Introduction}

The Navier-Stokes (Reynold's average) equation is an important model problem simplified from the complex fluid motion. Through the in-depth study of this model, we can understand and master the natural laws to promote the progress of natural science. However, the Navier-Stokes equation is a nonlinear partial differential equation, which is very difficult to solve. Only simple flow problems can get the exact solution, but most of the complex problems still need to have the j-solution by the discrete method. Therefore, the study of the j-solution of the equation is of great practical importance. The Navier-Stokes equation has been studied by many researchers and is very popular in the field of physics. Therefore, it is not feasible to provide insights into every aspect of it in detail. A few problems have been selected in this article for detailed discussion. Mathematically, the stable equation has one more nonlinear term than the existing Navier-Stokes equation. The nonlinear term makes the solution of the equation very difficult, and the calculation becomes very large and complex after discretization. At present, Reynolds average method is mainly used to solve the problems of fluid mechanics and other engineering problems.

Firstly, the instantaneous motion satisfying the dynamic equation is decomposed into mean motion and pulsating motion. Since the pulsation motion is completely irregular random motion, the only concern about it is that the contribution of pulsating motion to average motion is modelled by Reynold's stress term. Then, according to the theoretical knowledge of turbulence, experimental data or the results obtained from the direct numerical simulation, various hypotheses are formed for Reynolds stress, and various empirical plus semiempirical model equations are 
obtained so that the average Reynolds equation of turbulence can be ended up. According to the various assumptions of Reynolds stress, the turbulence model can be divided into the Reynolds stress model and Eddy viscosity model.

The transport equation of force and other second-order correlations go through a large number of calculations, so its application scope remains limited due to calculation complexity. Therefore, the Eddy viscosity model is widely used in engineering turbulence problems. At present, the mature turbulence models are based on the assumption of Eddy viscosity, which considers that the Reynolds stress tensor is proportionate to the average velocity gradient. On the basis of this assumption, many studies have been carried out by the researchers and it is found that there is nonexistence of a universal turbulence model for the theoretical derivation and analysis of a large number of experimental data.

In [1], the momentum source term has been modified to reflect the effect of the wind turbine on the wake velocity, and the turbulent model is modified by adding the turbulent energy term and dissipation rate source term to the standard equation. The addition is being made to coordinate the generation and dissipation of turbulent kinetic energy; the source term coefficient of dissipation rate is also modified to follow the parabolic distribution along the radial direction. The wind tunnel experimental data are selected to validate the model, and the wake flow field under tandem and staggered arrangement is analyzed. The results show that the velocity loss increases with the increase in the spacing, and the turbulence intensity of the wake decreases with the increase in the spacing. The effect of staggered arrangement on the turbulence intensity is greater than that on the velocity, and the turbulence intensity asymmetry appears in the downstream wake, which is the most serious concern when the transverse spacing is 1 , and the asymmetry phenomenon can still be observed when the transverse spacing is 4. In [2], the influence of the uncertainty of turbulence model coefficients on the transonic flow simulation of RAE2822 airfoil is studied by using the nonembedded polynomial chaos method. The uncertainty quantification results of integral (lift coefficient and drag coefficient) and local quantity (wall pressure, friction coefficient, and space Mach number distribution) of numerical simulation are analyzed in this paper. The influence of uncertainty of Carmen constant on numerical simulation is studied from the single input variable. Then, the influence of uncertainty of nine parameters in the Spalart-Allmaras model is considered. Finally, during the process of multivariable uncertainty quantification, the Sobol index is used to quantify the contribution of each input variable's uncertainty to the output uncertainty. Only RAE2822 transonic velocity is considered in this paper for evaluation.

In [3], ethylene cracking furnaces equipped with bottom burners and sidewall burners are widely used. Different combustion modes affect the turbulent flow state in the furnace. Considering the strong nonlinear coupling effect between turbulent flow and gas injection, combustion, and heat transfer in the cracking furnace, it is necessary to further test whether the influence law can be extended to other working conditions and situations. Therefore, it is very significant to explore the impact of different turbulence models in the cracking furnace/reactor coupling simulation for the precise design and optimization of the cracking furnace. The coupling simulation of a 100000-ton industrial Ethylene cracking furnace is carried out with different turbulence models. The simulation results of the three turbulence models are compared with the industrial data and multiple parameters such as velocity, temperature, and turbulence capacity in the cracking furnace. The results show that the realizable $\mathrm{K}-\varepsilon$ model is superior to the other two models in flame stability and reaction efficiency. This model is based on the realizable model. The results obtained from $\mathrm{K}-\boldsymbol{\varepsilon}$ turbulence equation in the heat flux and the temperature distribution of the outer wall of the furnace tube are closer to the actual working conditions.

In order to describe the fluid flow at Reynolds number, some terms of the complete Navier-Stokes equations are often omitted and simplified to a specific hydrodynamic equation. When the fluid flows along a mainstream direction, the viscous term along the mainstream direction is lost, and all the inviscid terms and the main viscous terms that can reflect the interaction between viscous flow and inviscid flow are retained. For example, when the fluid flows along the $x$-axis direction, then the Navier-Stokes equation can be obtained by omitting the second-order viscous term of $X$ and by retaining other second-order terms and inviscid nonlinear terms in the equation. The Navier-Stokes equation is essentially a hyperbolic parabolic partial differential equation system, which can be used to describe the two-dimensional fluid flow along the main flow direction ( $x$-axis direction). A large number of numerical calculations show that there is no large-scale separation in the mainstream direction especially for the fluid flow with Reynolds number. The numerical viscosity of the complete Navier-Stokes equation often conceals the real physical viscosity. The final numerical result is not the $\mathrm{j}$-solution of the Navier-Stokes equation, but the j-solution of the simplified Navier-Stokes equation. Therefore, only a simplified Navier-Stokes equation is required to solve the problem in the field of physics. The Navier-Stokes equation can describe the real fluid flow. Although it seems unreasonable to use the simplified Navier-Stokes equation to describe the separation zone in the flow direction, the large number of calculations show that there are local small separation areas in the Reynolds number flow along the main flow direction (such as front step, back step, separation bubble, compressed corner flow, and inlet passage flow field) where the simplified Navier-Stokes equation is used. Although the Navier-Stokes equation is greatly simplified to the complete Navier-Stokes equation, it can be used by the global relaxation method and the space advancing iteration method, which is capable enough of reducing the dimension of the discrete equations and the amount of calculation complexity.

However, there are still many areas in solving large-scale practical problems, but due to the accumulation of errors in the truncation method, there is a high degree of error accumulation in the calculation process of the Navier-Stokes method. This method will not be able to obtain the ideal solution due to the accumulation of errors in the truncation 
method degree. The dimension reduction simulation model that can be continuously calculated forward has important theoretical value and application prospects.

\section{Numerical Analysis Method}

At present, there are three kinds of direct numerical simulation methods, namely, direct numerical simulation method, Eddy simulation, and Reynolds average equation.

The direct numerical simulation method obtains the instantaneous flow field of fluid motion by directly solving the complete three-dimensional unsteady equation, which controls the fluid movement and obtains all the information of fluid flow. The greatest advantage of the direct numerical simulation method is that the equation controlling fluid motion is accurate, and the calculation error does not include model error, which is mainly caused by the method error space. It provides a direct solution of the unsteady Navier-Stokes equations; it is also capable of resolving the smallest eddies and time scales of turbulence in the flow. Secondly, it can provide all the information of every instantaneous flow in the flow field. With the rapid development of computer technology and computing technology, this method has become an effective method to solve flow problems. However, due to the existence of vortices with different sizes, the maximum scale can be equivalent to the characteristic length of the mean motion, while the minimum scale depends on the viscous dissipation velocity. Moreover, the ratio of the size increases with the increase in the number. The complexity of the turbulent flow brings great difficulties to the direct numerical simulation; it is usually required that the scale of the computational grid should reach the order of several powers. Such a scale of computational grid poses a great challenge to the speed and capacity of the computer. Therefore, at present, it is only limited to a simple flow with low Reynolds number and a very simple flow shape, which is not suitable for high Reynolds number flows.

The large Eddy simulation method (LES) refers to the simulation of the turbulent velocity field, which serves as a compromised method between direct simulation and equation method. As the turbulent motion is formed by the vortex motion of many different scale structures in the process of turbulent motion, the energy of the large-scaled structure vortex is directly provided by the average flow. The average flow is closely related to the initial conditions, boundary properties, and boundary shape of the flow. The shear correlation is highly anisotropic when the small-scale structural vortices are generated by the nonlinear interaction between large-scale structural vortices. The average flow plays the role of viscous dissipation and the energy is mainly transmitted by large-scale structural vortices. When the scale of vortices is smaller to a certain extent, it will not be affected by the external conditions of flow. In view of this property of turbulent motion, the equation is averaged in a small space domain, which is also called filtering. The purpose is to remove the small-scale structure vortices from the flow field and derive the equation satisfied by the large-scale structure vortex. The influence of the small-scale structure vortex on the large-scale structure vortex will appear in the large-scale structure vortex equation, which is called the subscale structure vortex equation. A lattice-scale model is used to simulate small-scale structural vortices, while the characteristics of large-scale structural vortices are obtained by numerically solving the filtered equations. Since the year shape theory has been applied to solve the simple hydraulic problems of channels, the method has gradually become one of the powerful tools to solve the hydrodynamic problems. In recent years, it has been developed vigorously to solve simple flow problems. The method is limited to solve simple flow problems and has not been exploited to solve complex problems. At present, time-averaged turbulence simulation is quite a good choice for engineering calculations. Reynolds average method has limitations in usage due to computer conditions.

At present, Reynolds average method is mainly used to solve the problems of fluid mechanics and engineering practices. Firstly, the instantaneous motion satisfying the dynamic equation is decomposed into mean motion and pulsating motion. Since the pulsation motion is a completely irregular random motion, the major concern about it is that the contribution of pulsating motion to average motion can be modelled by Reynolds stress term and then according to the theoretical knowledge of turbulence and experimental data; various hypotheses are made for Reynolds stress. On the basis of the hypothesis, various empirical and semiempirical model equations are obtained for ending up the average Reynolds equation of turbulence. According to the different assumptions of Reynolds stress, the turbulence model can be divided into the Reynolds stress model and Eddy viscosity model, where the turbulent stress model can be established directly. The transport equation of force and other second-order correlation quantities require a large number of calculations. Therefore, in order to carry out the numerical analysis of the $j$-solution of the Navier-Stokes equation, it is necessary to integrate multiple methods in terms of multimodel simulation analysis.

\section{Application Method of Navier-Stokes Equation}

Since the Navier-Stokes equation is nonlinear, it mainly directs the application of two sets of grids in the linearization of nonlinear problems. Whether it is theoretical analysis or numerical simulation, the realization becomes relatively complex, which is determined by the body of the equation. The equation has two forms: original variable form and stream function form:

$$
f=-v \cdot A u+(u, V) u+V p,
$$

where $(u, V)=0$, in $n,(3.3) u=0$. It can be a two-dimensional region or a three-dimensional region. Generally, the hybrid finite element method is used to solve the problem [4]. The schematic diagram of finite element simulation is shown in Figure 1.

Like the equation, the finite element discrete space selected by the finite element simulation should also satisfy the discrete conditions. 


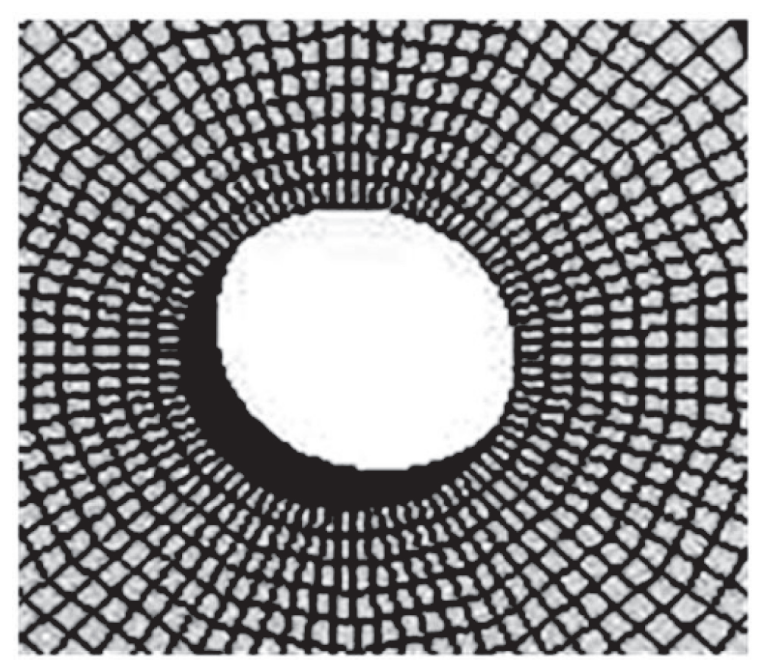

Figure 1: Schematic diagram of finite element simulation.

$$
(9 h, V, V h,)_{\text {BollihtlQn }}, \leq S_{\text {upVgh }} \in Q_{n} \cdot U_{h} \in V h_{l l v h l l v h} .
$$

Since the flow function $V h_{l l v h l l v h}$ does not exist in threedimensional space, the flow function form of the NavierStokes equation is only suitable for the two-dimensional case. Then, there exists a stream function $(x, y)$ in twodimensional space, which satisfies the following conditions:

$$
O \psi 8 \psi U 1=-8 y \cdot U 2=8 x \text {. }
$$

Such a stream function is uniquely determined by $u$ in the case of a constant difference. If we use the stream function as the function to find the solution of $j$, then the incompressible condition is automatically satisfied:

$$
V . u=8 u 1+8 u 2+8(x, y) \psi \cdot a \psi=(8 x, a y)+(8 y, O x)=0 .
$$

By cutting off variable $p$ in the original variable form (3.3), the corresponding stream function expression can be obtained as shown in

$$
v_{o} \cdot 2 \psi-\psi \cdot y_{Q} \cdot \psi \cdot x+\psi \cdot x_{o} \cdot \psi_{y}=\text { curlf, }
$$

where, in $n, \varphi=0$; on $8 \mathrm{~S} 2, x_{o}=0$; on $p+80, O_{n}=0, n$ represents the unit normal vector of $N$. The two grid methods are suitable for both schemes. In the above formulas, two sets of grid methods are applied to the Navier-Stokes equation in the form of stream functions, and the corresponding theoretical analysis and numerical experiments are given.

In this article, we mainly discuss the application of two sets of grid methods to the Navier-Stokes equations in the form of primitive variables. In this respect, the research studies are available in existing literature, and there will be more research endeavours on this aspect in the future. There is always a need for a balance; that is, there is a need for the proportional relationship between coarse and fine grids as much as possible to speed up the calculations. It is assumed that the Reynolds number Re is relatively smaller without considering the large Reynolds number (since $v$ is very small). At least for now, the Reynolds number, which can achieve a good calculation effect, is only about ten thousand orders of magnitude. It is still a great challenge to calculate the Navier-Stokes equation with a large Reynolds number.

$$
Y=X x_{Q}
$$

Among them,

$$
X=(H(2)) d
$$

where $d$ is the dimension of space.

$$
Q=L_{2}(n) \text {. }
$$

The variational problem corresponding to the above formula is as follows:

$$
Q=L_{2}(u)
$$

Then, equation (10) is applied:

$$
(u, p) \in Y \text {. }
$$

Then, the following is met:

$$
a(u, v)+b(u, u, v)-(p, V . v)+(q, V . u)=(f, v), \quad V(0, q) \in Y,(3.4)
$$

Here, the bilinear form $a(u, v)$ and the trilinear form $b$ $(u, w, v)$ are defined as follows:

$$
\begin{gathered}
a(u, v)=(v V u . V u) \cdot \mathrm{d} x, \\
b(u, w, v)=u . V \cdot(w, \mathrm{~d} x, V u,) .
\end{gathered}
$$

Among them,

$$
v, w \in X
$$

In this way, the discrete variational problem can also be expressed as follows:

$$
\left(u_{n}, V_{h}\right) \in Y_{h} .
$$

So,

$$
(v, q) \in Y_{h} .
$$

The following equation is satisfied: 


$$
a\left(u_{h}, v\right)+b\left(u_{n}, u_{h}, v\right)-\left(P_{h}, V v\right)+\left(q, V \cdot u_{n}\right)=(f, v) .
$$

Obviously,

$$
Y_{h}=X h_{x} \cdot Q_{h}
$$

In order to show the difference, we take the discrete solution of $h$ in the subscript position as the $j$-solution obtained by solving the nonlinear equation directly on $Y_{h}$, and the discrete solution of $h$ in the superscript position mentioned below is the j-solution obtained by two sets of grid methods. For this term $Y_{h}$, its variational form is not only $b$ $(u, u, w)$ mentioned here, which is also known as convective form. There are two other types of symmetry, namely,

$$
b_{2}(u, U, w)=f_{q}\left[\left(u, V_{v}, w\right)-\left(u, V_{w}, v\right)\right] \mathrm{d} x .
$$

The conservation forms are as follows:

$$
b(u, v, w)=-f\left(q_{u}, V_{w}, v\right) \mathrm{d} x .
$$

There is no significant difference in the properties of these three forms, so we will only discuss the convective form here and later.

\section{Partition of Multiblock-Structured Grid and Coarse plus Fine Grid in the Form of Flow}

In order to reduce the difficulty of grid generation for complex geometric shapes, the partition strategy is used in usual. The partition is needed to decompose the whole irregular shape region into regular subregions and to generate the computational grid independently on each subregion. There are two ways to divide the whole grid region, namely, overlapping partition and splicing partition. There are overlapping parts in the subregion, and the generated grid is also termed a nested grid. In the splicing partition, there is a common interface between the subregions. The subarea grid can be divided into multiple blocks with a common interface to generate the partitions separately. Multiple block grids can be merged into a single grid. If the points on the interface between the grid blocks and blocks are one-to-one correspondingly, then the grid lines across the interface are continuous, followed by generalized splicing, and continuous splicing is the most difficult to achieve. In terms of the difficulty level of the grid generation, the nested grid is the easiest way to generate the grid. However, in terms of the algorithm implementation of flow solver, the continuous splicing grid is the simplest one, the generalized splicing grid has more complexity in generation, and the nested grid is the most complex among the three methods. There are two strategies for grid block: one is to acquire the interface between blocks to be a complete grid edge interface and the other is to allow a certain interface of a grid to form an interface with multiple grid blocks. In this paper, the second blocking strategy has been used to generate multiblockstructured grids with continuous splicing.

Therefore, the first thing is to define two coarse and fine meshes of length $h$ and $H(H>h>0)$ and then construct a conforming finite element space, which is denoted as $\left(X_{H}, Q_{H}\right),\left(X_{H}, Q_{H}\right)$ respectively. It is assumed that the fine grid space $\left(X_{H}, Q_{H}\right)$ is subdivided by the grid coordinates of the coarse grid space $\left(X_{H}, Q_{H}\right)$ in Figure 2:

Our assumption is not necessary for the algorithm or convergence theorem, but it can make the analysis process easier. Under this assumption, the equation is written as follows:

$$
\left(X_{H}, Q_{H}\right) c>\left(X_{H}, Q_{H}\right) .
$$

It is assumed that this space has the approximation property of piecewise polynomials:

$$
(u, p) \in Y \cap\left[H_{k}+\left(S_{2}\right) \mathrm{d} x \cdot H *(Q)\right] .
$$

In semidiscrete form, $K$ is a polynomial coefficient, space is not discrete, and only time is discrete for simplicity. The second step of the projection algorithm is to project the intermediate velocity onto space with zero divergences. The projection algorithm mainly separates the viscous term from the incompressible term (divergence is zero).

However, when dealing with the far-field boundary, it is necessary to reflect the feature where the external field disturbance wave will not be reflected back to the internal field. In other words, the far-field boundary treatment should bifurcate the problem into an unmanned boundary layer and an unmanned boundary. The numerical calculation of the reflection should be carried out using time from the uniform flow field. Since the boundary conditions of the wall must be coincided with the surface of the object, it can be assumed that the disturbance waves will be striking from the object surface, and if the far-field boundary of the grid element is transferred from layer to layer, then the reflection of disturbance wave could be reduced to a considerable extent. The accuracy of the result will be greatly reduced in the latter case and the convergence rate of the steady solution will also be slowed down. The difference between the solution of the nonconvergent equation and the Euler equation may be embodied in the mathematical equation, the viscous term at the right end is also included, and then different boundary conditions are handled. If the boundary of the field is far enough, the velocity change will be very small in the far-field processing. Therefore, according to the different schemes, the projection method can be divided into three types: pressure modified correction scheme, velocity correction scheme, and harmonic splitting scheme.

In order to study the order of the scheme on time, we introduce the norm about time $[5,56,58]$ : given a Banach space $X$, whose norm is expressed as $\mathrm{Ix}$, a continuous function is $u$ : $[0,7] \longrightarrow X$, and two real numbers are $p>0$, $a>0$. For the time step $k>0$, let $t_{n}=n_{k}$, where $n=0, \ldots, M$; if there is a constant $C$, the following formula holds for all $k$ :

$$
\frac{\left(2 k l_{u}\left(t_{u}\right)-u\right) 1}{l_{x} \leq C_{k}} .
$$

A finite sequence approximates $u$ in IP $(X)$ of order a. When $u$ " $n=0, \ldots ., M$, the order " $a$ " approximates $u$. 


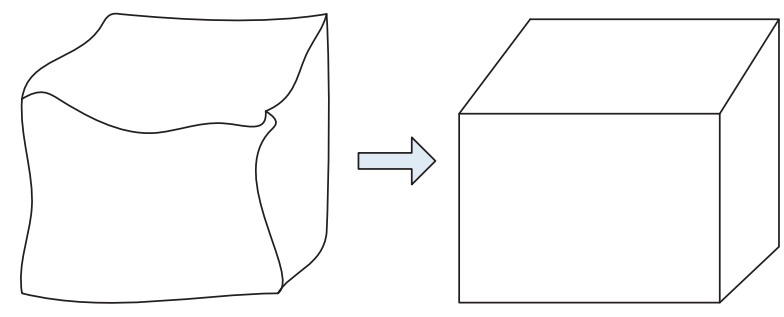

Coordinate transformation
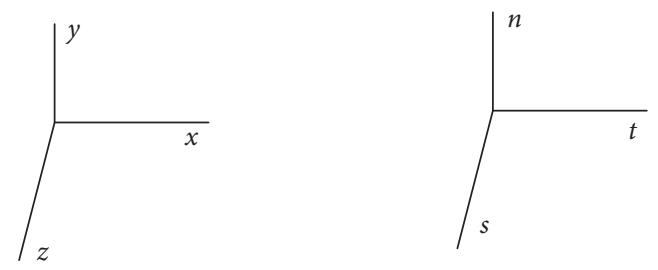

FIGURE 2: Grid coordinate subdivision diagram.

$$
\left|l_{u}\left(t_{u}\right)-u^{\prime \prime} l_{x}\right| \leq C_{k} \cdot V_{n}=M
$$

In this paper, the boundary conditions and the initial conditions of the solution of the problem are discussed. The degree equation is the governing equation of all viscous flows. In the process of solving specific flow problems, the corresponding boundary conditions and initial conditions are required to be added reasonably. Since this paper solves the steady flow problems, the initial conditions can be given arbitrarily in theory [5]. Therefore, one of the main problems in solving the equations in this paper is the numerical discretization of boundary conditions; if the boundary conditions are not handled properly, it will not only cause large errors in the calculation results [6] but also make the calculation process unstable, which may lead to the early convergence. The values of $C$ are different in different places. They may depend on the data $f, u_{o}, t$, viscosity coefficient $V$, and region and continuous solution $u$, but have nothing to do with time step $k$ and space step $h$.

We have considered the $\mathrm{j}$-solution of the corresponding problem as the initial iteration value. Because of its small scale, it takes lesser time than solving large linear systems on fine grids. In fact, we can ascertain formula (12).

$$
\left|V \cdot p_{n}+(1, n)\right| a_{n}=0 .
$$

This condition is added in addition, and there is no such requirement in itself. Therefore, the artificial Neumann boundary condition brings in a numerical boundary layer $[7,8]$ so that the velocity $u$ in $h$ norm and $p$ in I2 norm cannot reach the first order. For this scheme, there is an inherent splitting error $k$, so for the operator $8 t-v$, the use of a higher-order splitting scheme cannot improve any effect. It is also noted that in the first step, there is no pressure gradient $[9,10]$, so further improvement is needed.

\section{Turbulence Model-Based Simulation}

Compared with other methods, the turbulence model has the advantages of simplicity, easy implementation, and less strict grid requirements. Therefore, this paper adopts the turbulence model. The grid-centered finite volume method is used to directly apply the integral equation to each grid element. Then, the flux term is calculated by the interpolation and difference method, and the integral equation is transformed into the integral equation. It is used to solve the algebraic discrete equations with the central physical quantity of the grid element as the unknown quantity $[11,12]$.

The turbulence model is based on Reynolds average motion equation and pulsating motion equation, relying on the combination of theory and experience. The main task of this paper is to establish a set of closed equations describing the mean turbulent flow. Its main task is to establish a calculation method with sufficient accuracy and general applicability for predicting Reynolds stress and scalar transport term. According to the different assumptions of Reynolds stress in the turbulent flow process, the turbulence model can be divided into the Reynolds stress model and Eddy viscosity model. The Reynolds stress model directly establishes the turbulent stress. In the Eddy viscosity model, the second-order correlation of velocity fluctuation is expressed as the product of average velocity gradient and turbulent viscosity coefficient:

$$
-8 \delta \cdot P_{u} \max (u, l)-p_{k},
$$

where $l$ is the turbulent viscosity coefficient and $\delta$ is the Kronecker operator.

At present, there are three kinds of spatial discretization methods for numerical simulation equations: finite difference method, finite volume method, and finite element method. The first two methods are commonly used in the structural grid. The finite difference method replaces the partial derivative term of differential equation with difference quotient on discrete grid points. The main idea of the finite difference method is to apply the integral control equation of fluid mechanics to every element in such a way that the solution of the integral equation is transformed into the solution of an algebraic equation or ordinary differential equation. Then, the time marching scheme is used to iterate. Finally, the stable solution of the flow field is obtained on each control volume element. Generally speaking, the resolution of the finite difference method for the boundary layer is higher than that of the finite volume method, while the finite volume method is more effective for multiple blocks. The finite volume method has the advantages of a simple algorithm, easy implementation [13, 14] and less strict grid requirements. In this paper, the grid-centered finite volume method is adopted, and the integral equation is directly applied to each grid element. Then, the interpolation and difference methods are used. The flux term is calculated, and the integral equation is transformed into an algebraic discrete equation system with the central physical quantity of the grid element as the unknown quantity. After spatial discretization, the equation is transformed into a first-order ordinary differential equation system about time on each grid cell. These ordinary differential equations are solved along time by explicit or implicit methods. The practical and 
effective method to solve the steady flow problem is not only simple and easy to implement but also occupies less memory. It can also adopt a variety of accelerating convergence measures, for example, local variable time step, implicit residual smoothing, baking damping, and multigrid. However, the explicit method has its inherent defects, poor stability, and small time step, but the implicit time marching method can take a larger time step in the whole flow field with good stability and high computational efficiency. The implicit time-stepping method consumes a lot of computer resources, especially computer memory. Therefore, the two methods should be served as an input into the simulation environment to learn from each other. In order to shorten the calculation time, the number of closed equations (the number of differential equations needed to establish the turbulence model) involved in the turbulent viscosity coefficient can be directly solved. Firstly, the pressure correction scheme is set. The first step is given, which is omitted here $[15,16]$. The former is a second-order form, and the latter is a first-order form. Then, in the second step, the obtained intermediate velocity is projected into space with zero divergences. The specific format is as follows:

Order:

$$
u=\frac{u_{1}}{\left(O_{\mathrm{in}}+t_{1} 1-u\right)-v O_{\mathrm{in}}+i+\left(1, V i^{\prime \prime}\right)+1}=f\left(T_{p}^{\prime \prime}\right) .
$$

By solving elliptic partial differential equations and algebraic methods, a hypercube coarse grid with 10000 grid points is generated using the above grid scheme [17]. The following requirements are considered when generating the coarse grid.

(1) The distance between the first grid and the surface is $1 \times 10^{-5}$.

(2) In order to avoid overstretching of mesh near the object surface [18], the order of change of normal mesh step is $O$.

(3) On the basis of the coarse grid, the normal direction grid points of the object surface are kept unchanged, the chord and spanwise grid points are appropriately increased, the length-width ratio of the grid is improved, and the hypercube medium density grid with ten thousand grid points is generated. On the basis of the medium grid $[19,20]$, the distance from the first grid to the object surface is reduced from fire to fire. When the grid is smaller to other two directions, increase the number of normal grid points on the object surface, improve the length-width ratio of the mesh to avoid excessive stretching of the mesh near the object surface [21], and then form a hypercube fine grid, referred to as fine grid, with a scale of 10000 grid points. So far, the simulation construction of the Reynolds hypothesis fine grid after turbulence improvement is completed, as shown in Figure 3.

Figure 4 shows the Reynolds assumption of $J$ component of velocity $u$ in the fine grid simulation.

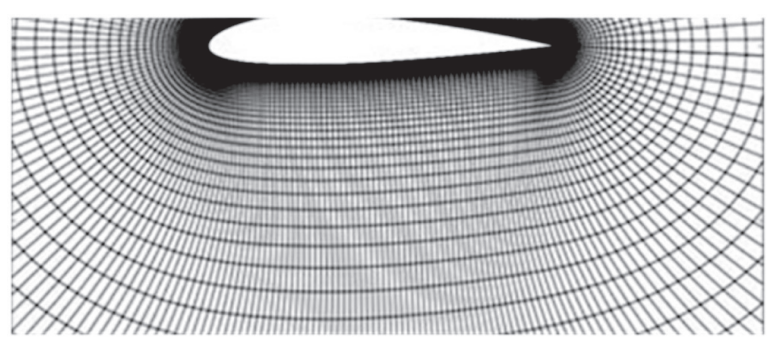

Figure 3: Simulation results of Reynolds hypothesis fine grid.

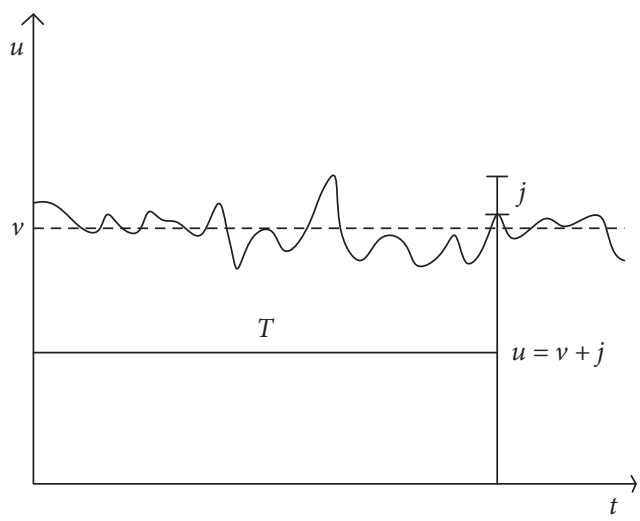

FIgURE 4: Reynolds assumption of $\mathrm{j}$ component of velocity $u$.

\section{Conclusion}

In this paper, different numerical analysis methods are discussed, and the conservation form is defined. According to the computational properties of the three forms, the application of two sets of grid methods on the Navier-Stokes equation has been studied. The coarse and fine grids are defined in the flow form of $j$-solution. At the same time, the $\mathrm{j}$-solution of the corresponding problem is taken as the initial iteration value, and the numerical analysis of the j-solution of Navier-Stokes equation is completed. Firstly, the automatic grid generation technology of high-quality grid generation method is carried out to improve the grid quality and shorten the grid generation period. Then, the turbulence model equation is applied. Secondly, the existing turbulence model equations under various flow conditions are evaluated scientifically. In order to improve the simulation accuracy of turbulence, a high-order algorithm for solving the model equation and turbulence model equation has been developed. In order to solve the problem of grid dependency, reduce the numerical error, and improve the computational efficiency, the proposed method has been deployed. The results show that the true method can avoid mesh degradation skilfully, and the generated mesh has the characteristics of smoothness, orthogonality, and controllability, which can eventually improve the calculation accuracy.

\section{Data Availability}

The data are available on request to the corresponding author. 


\section{Conflicts of Interest}

The authors declare that they have no conflicts of interest.

\section{References}

[1] H. L. Ren, X. D. Zhang, and S. Kang, "Study of actuator disc method based on improved turbulence model," Journal of Chinese Society of Power Engineering, vol. 39, no. 1, pp. 65-71, 2019.

[2] H. Zhao, X. Z. Hu, J. Zhang, J. T. Chen, and M. S. Ma, "Effects of uncertainty in turbulence model coefficients on flow over airfoil simulation," Acta Aeronautica ET Astronautica Sinica, vol. 40, no. 6, pp. 68-78, 2019.

[3] C. Z. Ni, W. L. Du, and G. H. Hu, "Impact of turbulence model in coupled simulation of ethylene cracking furnace," CIESC Jorunal, vol. 70, no. 2, pp. 450-459, 2019.

[4] V. Jagota, A. P. S. Sethi, and K. Kumar, "Finite element method: an overview," Walailak Journal of Science \& Technology, vol. 10, no. 1, pp. 1-8, 2013.

[5] D. A. Di Pietro and S. Krell, "A hybrid high-order method for the steady incompressible navier-stokes problem," Journal of Scientific Computing, vol. 74, no. 3, pp. 1677-1705, 2018.

[6] T. Masahisa and U. Shinya, "An exactly computable lagrange-galerkin scheme for the navier-stokes equations and its error estimates," Mathematics of Computation, vol. 87, no. 309, pp. 39-67, 2017.

[7] H. Qiu, "Two-grid stabilized methods for the stationary incompressible navier-stokes equations with nonlinear slip boundary conditions," Applied Mathematics and Computation, vol. 332, pp. 172-188, 2018.

[8] E. Feireisl, Y. Lu, and A. Novotný, "Weak-strong uniqueness for the compressible navier-stokes equations with a hardsphere pressure law," Science China Mathematics, vol. 61, no. 11, pp. 2003-2016, 2018.

[9] P. Antonelli and S. Spirito, "On the compactness of finite energy weak solutions to the quantum navier-stokes equations," Journal of Hyperbolic Differential Equations, vol. 15, no. 1, pp. 133-147, 2018.

[10] A. Decaster and D. Iftimie, "On the asymptotic behaviour of 2D stationary navier-stokes solutions with symmetry conditions," Nonlinearity, vol. 30, no. 10, pp. 3951-3978, 2017.

[11] G. J. Gassner, A. R. Winters, F. J. Hindenlang, and D. A. Kopriva, "Correction to: the BR1 scheme is stable for the compressible navier-stokes equations," Journal of Scientific Computing, vol. 77, no. 1, pp. 201-203, 2018.

[12] S. Reuther and A. Voigt, "Solving the incompressible surface navier-stokes equation by surface finite elements," Physics of Fluids, vol. 30, no. 1, Article ID 012107, 2018.

[13] J. Brezina, E. Feireisl, and A. Novotny, "Stability of strong solutions to the navier-stokes-fourier system," SIAM Journal on Mathematical Analysis, vol. 52, no. 2, pp. 22-26, 2018.

[14] P. Maremonti and S. Shimizu, "Global existence of solutions to 2-D navier-stokes flow with non-decaying initial data in half-plane," Journal of Differential Equations, vol. 265, no. 10, pp. 5352-5383, 2018.

[15] J. Li and X. M. Liang, "Artificial fish swarm algorithm convergence speed improvement optimization simulation," Computer Simulation, vol. 35, no. 1, pp. 232-238, 2018.

[16] M. Higaki, Y. Maekawa, and Y. Nakahara, "On stationary navierstokes flows around a rotating obstacle in two-dimensions," Archive for Rational Mechanics and Analysis, vol. 228, no. 2, pp. 603-651, 2018.
[17] J. Neustupa and H. Al Baba, "The interior regularity of pressure associated with a weak solution to the navier-stokes equations with the navier-type boundary conditions," Journal of Mathematical Analysis and Applications, vol. 463, no. 1, pp. 222-234, 2018.

[18] X.-G. Yang, L. Li, and Y. Lu, "Regularity of uniform attractor for 3D non-autonomous navier-stokes-voigt equation," Applied Mathematics and Computation, vol. 334, no. 12, pp. 11-29, 2018.

[19] Y. L. Liu and P. Zhang, "On the global well-posedness of 3-D axi-symmetric navier-stokes system with small swirl component," Calculus of Variations \& Partial Differential Equations, vol. 57, no. 1, 2017.

[20] B. Carrillo, X. Pan, Q. S. Zhang, and N. Zhao, "Decay and vanishing of some $\mathrm{D}$-solutions of the navier-stokes equations," Archive for Rational Mechanics and Analysis, vol. 237, no. 4, pp. 1383-1419, 2020.

[21] R. Yang and X.-G. Yang, "Asymptotic stability of 3D navier-stokes equations with damping," Applied Mathematics Letters, vol. 116, Article ID 107012, 2021. 